\title{
Predição de Tamanho de Código utilizando Benchmarks Sintetizados: Um Estudo de Caso
}

\author{
André Felipe Zanella ${ }^{1}$, Anderson Faustino da Silva ${ }^{1}$ \\ ${ }^{1}$ DIN - Universidade Estadual de Maringá (UEM) - Maringá - PR - Brasil \\ aft.zanella@gmail.com, anderson@din.uem.br
}

\begin{abstract}
One of the main issues preventing Deep Neural Networks from becoming mainstream on compiler optimization is the difficulty of creating high quality training datasets. Real world programs are usually large and complex, and due the lack of benchmarks, predictive compilation models usually fails to generalize due the vast space of programs. Recent efforts from the community to mitigate this problem have resulted on open-source benchmark synthesis tools capable of generating thousands or millions of synthetic benchmarks. However, these code hardly match in terms of similarity with hand coded benchmarks. This paper aims to evaluate the performance of AnghaBench benchmarks, a prominent suit of synthetic $C$ files for predictive compilation, in a machine learning task. We compare the performance of these benchmarks against applications from the LLVM test suite, using a LSTM model to predict the code size of a program provided by a optimization sequence without the need to compile it. The experimental results indicates that, in some domains, the synthetic benchmarks performs equally like the real applications, but does not outperforms it.
\end{abstract}

Resumo. Um dos principais problemas que impedem Redes Neurais Profundas se tornarem predominantes para otimização de compiladores é a dificuldade de criação de conjuntos de dados de alta qualidade. Benchmarks reais geralmente são programas grandes e complexos, e por não serem abundantes, modelos de compilação preditiva geralmente apresentam dificuldades de generalização devido ao vasto espaço de programas. Esforços recentes da comunidade para mitigar este problema resultaram em ferramentas para síntese automática de benchmarks, capazes de gerar milhares ou milhões de programas sintéticos. No entanto, esses códigos dificilmente coincidem em termos de similaridade com benchmarks codificados manualmente. Este artigo tem como objetivo avaliar o desempenho dos benchmarks AnghaBench, uma proeminente suite de programas $C$ sintetizados para compilação preditiva, em um problema de aprendizagem de máquina. Comparamos o desempenho desses benchmarks com aplicações provenientes da suíte de testes LLVM, utilizando um modelo LSTM para prever o tamanho de código proporcionado por uma sequência de otimizações, sem a necessidade de compilar o programa. Os resultados experimentais indicam que, em alguns domínios, benchmarks sintéticos são equiparáveis, mas não superam aplicações reais.

\section{Introdução}

A última década foi agraciada com grandes avanços envolvendo aplicações de Redes Neurais Artificiais (do inglês Artificial Neural Network ou ANNs) para mitigação de diversos 
problemas, principalmente graças ao suporte de aceleradores de hardware especializados. O método de aprendizagem tem sido a abordagem padrão em diversas tarefas, incluindo, mas não limitado à, Análise de Imagens [Wei et al. 2019], Processamento de Linguagem Natural [Otter et al. 2021] e Sistemas de Recomendação [Zhang et al. 2019]. ANNs e outros métodos de aprendizagem de máquina tem sido adotados pela comunidade de compiladores, em geral, para encontrar boas sequências de otimização ${ }^{1}$ que superem planos pré-definidos por compiladores como GCC 2]e LLVM [Lattner and Adve 2004].

Apesar da adoção pela comunidade, a pesquisa em otimização de compiladores auxiliada por ANNs ainda é modesta quando comparada as áreas de pesquisa já citadas. Um grande limitante para uma adoção mais ampla é a dificuldade de construção de conjuntos de dados de alta qualidade, i.e., abrangentes conjuntos de programas para treinamento que englobem significativamente as características de diversos domínios de aplicações. Tal fato ocorre devido a escassez de benchmarks disponíveis e a grande demanda por dados exigida por experimentos envolvendo ANNs [Cummins et al. 2017].

Síntese de benchmarks é uma abordagem promissora para mitigar o problema da escassez de dados. Visando compilação preditiva, o método consiste em gerar de forma algorítmica, grandes quantidades de benchmarks (milhares ou milhões) que encapsulem diversas propriedades semânticas e se assemelhem a benchmarks reais [Cummins et al. 2017]. No entanto, gerar código sintético que seja similar a programas reais é uma tarefa difícil. Diversos problemas devem ser mitigados dependendo da abordagem de geração, como por exemplo, inferência de tipos [da Silva et al. 2021] e treinamento de ANNs [Cummins et al. 2018]. Consequentemente, estes programas podem não corresponder fielmente a uma carga de trabalho convencional.

Um promissor conjunto de benchmarks sintéticos com mais de 1 milhão de amostras foi recentemente disponibilizado por Silva et al [da Silva et al. 2021]. O conjunto denominado AnghaBench, foi gerado a partir de um framework composto por um mecanismo de mineração de código em repositórios de código aberto na plataforma Git Hub ${ }^{3}$, um extrator de funções de aplicações, e um motor de inferência de tipos. Os benchmarks gerados são arquivos $C$ compiláveis, i.e., passíveis de geração de código objeto. Portanto, são programas direcionados para o estudo de propriedades estáticas e otimização de tamanho de código.

Os autores avaliam seus benchmarks sintéticos em comparação com outros geradores automáticos de programas, como Deepsmith [Cummins et al. 2018] e LDRGen [Barany 2018b] em termos de similaridade com programas reais e, geração de sequências de otimização para redução de código. Em contraste, este trabalho busca avaliar o desempenho de programas AnghaBench em um sistema de compilação preditiva baseado em Redes Neurais Recorrentes (LS TM) [Hochreiter and Schmidhuber 1997]. A heurística de compilação consiste em determinar a quantidade de instruções de uma representação intermediária (IR) LLVM não otimizada, quando uma sequência de passos de otimização é aplicada. Avaliamos a acurácia do modelo em diferentes domínios de programas e comparamos os resultados obtidos com benchmarks reais. Nossos experimen-

\footnotetext{
${ }^{1}$ Um plano, ou sequência de otimização, é um conjunto de passos de transformações aplicados pelo compilador durante o processo de geração de código final.

${ }^{2}$ https://gcc.gnu.org/

${ }^{3}$ https://github.com/
} 
tos indicam que o modelo treinado com programas reais detêm os melhores resultados. Providenciamos uma discussão com relação as possíveis causas, em termos da modelagem do problema, heurística de compilação e conjuntos de dados.

\section{Trabalhos Relacionados}

Atualmente, a comunidade de Linguagens de Programação e Compiladores dispõem de algumas ferramentas geradoras de benchmarks. Alguns exemplos são os geradores de kernels OpenCL, tais como CLgen [Cummins et al. 2017] e DeepSmith [Cummins et al. 2018], e de programas C, LDRGen [Barany 2018b], CSmith [Yang et al. 2011] e AnghaBench [da Silva et al. 2021].

Código sintético já foi experimentado como base para compilação preditiva em trabalhos anteriores [Barany 2018a] [Hashimoto and Ishiura 2016] [Huang et al. 2020], no entanto, estes não levantaram a questão comparativa da performance com relação a outros conjuntos. Um trabalho similar em natureza a nossa proposta é realizado por Goens et al. [Goens et al. 2019]. Os autores propõem um estudo de caso em que avaliam artefatos gerados pela ferramenta CLgen em comparação aos benchmakrs utilizados como base de dados pela ferramenta. O problema abordado em questão é o mapeamento de dispositivos (GPU / CPU) utilizando aprendizagem de máquina. Similarmente à AnghaBench, CLGen utiliza uma base de programas coletados de repositórios de código aberto, porém, diverge na metodologia generativa, fazendo uso de redes LSTM. Os resultados indicam que as aplicações geradas não apresentam performance positiva em relação a aplicações reais.

Os autores da suíte AnghaBench avaliam os artefatos produzidos no tocante a: (1) similaridade com benchmarks reais; (2) predição do impacto de sequências de otimização; e (3) base de treinamento para Aprendizagem de Máquina. Em todas as questões AnghaBench é avaliado com relação à outras coleções de programas sintéticos, apresentando vantagens sobre estes. Em (3), os autores avaliam a suíte em relação a suíte de testes LLVM em uma heurística de criação de sequências para redução de código. Nossa proposta difere, pois, realizamos testes em benchmarks reais de diferentes domínios em um sistema baseado em LSTM.

Outra questão pertinente a este trabalho é a geração de código reduzido. Como aponta Ashouri et al. [Ashouri et al. 2018], o problema de predição de tamanho de código passou a ser uma preocupação secundária com o avanço de sistemas de armazenamento. No entanto, pesquisas recentes trazem a tona esta questão novamente. Trofin et al. [Trofin et al. 2021] adotam Aprendizagem por Reforço para decidir quando se fazer inline em uma chamada, alcançando códigos até $7 \%$ menores em comparação à clang -Oz. Heo et al. [Heo et al. 2018] também fazem uso do mesmo paradigma em combinação com técnicas de depuração para reduzir códigos intermediários LLVM. Silva et al. [Silva et al. 2021] realizaram um estudo sistemático sobre o espaço de transformações para redução de código e propõem uma metodologia para criação de sequências especializadas para tal tarefa. A metodologia proposta pelos autores foi utilizada no presente trabalho para criação de sequências de transformações. Ortogonalmente, novas transformações para redução de código foram pospostas por Rocha et al. [Rocha et al. 2019, Rocha et al. 2020]. 


\section{Descrição do Problema e Objetivos}

Encontrar bons planos de compilação, isto é, sequências de transformações que providenciem melhorias no código final é uma tarefa difícil devido ao vasto espaço de exploração. A busca exaustiva por um plano de compilação é muitas vezes inviável, pois, é necessário a compilação e, dependendo do objetivo, a execução da aplicação para avaliação de sua performance. Devido a isso, é desejável, em muitos casos, realizar compilação preditiva, a fim de se conhecer de antemão o desempenho que uma sequência pode proporcionar sem a necessidade da compilação e execução da aplicação propriamente dita [Ashouri et al. 2018].

Neste trabalho, modelamos o problema de predição de tamanho de código como um problema de regressão. A métrica utilizada para medição é o número de instruções do código intermediário LLVM. Dado um programa IR não otimizado, denotado $P_{\text {noopt }}$, e uma sequência de transformações $S$, desejamos encontrar uma função de predição $R$, tal que $R\left(P_{\text {noopt }}, S\right)$ forneça com alta probabilidade a estimativa do número de instruções LLVM do programa $P_{S}$, isto é, o programa $P_{\text {noopt }}$ compilado com a sequência $S$. Para escolha do modelo de aprendizagem profunda, partimos da premissa de que linguagens de programação devem ser abordadas com os mesmos princípios e técnicas utilizados para Processamento de Linguagem Natural. Para atender tal requisito, utilizamos Redes Neurais Recorrentes LSTM em camadas e vetores densos de alta dimensão, também chamados de embeddings, para codificação numérica das aplicações, onde cada instrução LLVM é um vetor denso de 200 elementos.

Com o problema de compilação e modelo de Rede Neural definidos, a tarefa passa a ser avaliar a performance dos benchmarks sob estas condições. Avaliamos duas classes de benchmarks AnghaBench, a primeira contém arquivos com uma única função e a segunda contém arquivos com múltiplas definições de funções. Como base comparativa, treinamos nosso modelo com benchmakrs reais coletados da suíte de testes da infraestrutura LLVM. As predições foram realizadas em aplicações novas, dentre estas, foram selecionados benchmarks desenvolvidos para avaliação de sistemas embarcados. A justificativa para tal escolha está no fato de dispositivos embarcados serem os que mais se beneficiam de código pequeno. Também construímos um conjunto de dados para testes formado exclusivamente por arquivos contendo uma única função. O principal objetivo deste conjunto é avaliar a classe de dados AnghaBench com uma única função. Por fim, realizamos a avaliação em uma partição de programas previamente selecionados da suíte LLVM.

\section{Materiais e Métodos}

Como discutido nas seções anteriores, o principal objetivo deste trabalho é avaliar conjuntos de benchmarks sintéticos no problema de compilação preditiva. Para tal, definimos diferentes conjuntos de dados, uma representação de programas, construímos sequências de otimização e uma heurística de compilação.

Conjuntos de Dados e Estatísticas. Os dados que compõem os conjuntos de treino e teste foram coletados da suíte AnghaBench, da suíte de teste da infraestrutura LLVM e dos benchmarks Mibench $4^{4}$ e Coremark-Pro 5 . Separamos os conjuntos de dados em classes, as quais passaremos a nos referir com as seguintes nomenclaturas:

\footnotetext{
${ }^{4}$ http://vhosts.eecs.umich.edu/mibench//

${ }^{5}$ https://www.eembc.org/coremark-pro/
} 
- Angha15: Dentre os arquivos de funções AnghaBench encontramos as 15.000 maiores funções e selecionamos aleatoriamente 2.000 funções para compor o conjunto de dados.

- AnghaW: AnghaBench também contém programas completos, com aproximadamente 15.000 amostras. Seguimos a mesma metodologia e selecionamos aleatoriamente 2.000 amostras para este conjunto.

- LLVM Suíte: Classe composta por 218 aplicações de diversas conjuntos de benchmarks. Dentre estes, particionamos de forma aleatória 178 aplicações para treino e 40 aplicações para teste.

- Embarcados: Esta classe compreende as suítes Coremark-Pro contendo 9 aplicações e Mibench com 19 aplicações.

- Funções: Extraímos funções individuais de cada suíte da classe Embarcados e selecionamos os 300 maiores arquivos (nr. de instruções LLVM) de cada um quando compilados com opt - -00 . Denotaremos os conjuntos da classe como Coremark-f e Mibench-f.

Dentre as classes definidas, utilizamos para testes os programas Embarcados, Funções e uma partição da classe LLVM Suíte. Adicionalmente, utilizamos para treinamento as combinações Angha 15+LLVM e AnghaW+LLVM. As Tabelas 1 e 2 sumarizam as estatísticas com relação a quantidade de instruções dos conjuntos.

Tabela 1. Estatísticas dos conjuntos de dados utilizados para treinamento e teste.

\begin{tabular}{|c|c|c|c|c|c|c|c|c|c|}
\hline \multirow[b]{2}{*}{ Instruções } & \multicolumn{3}{|c|}{ Angha15 } & \multicolumn{3}{|c|}{ AnghaW } & \multicolumn{3}{|c|}{ LLVM Suíte (Treino) } \\
\hline & Min & Média & Max & Min & Média & Max & Min & Média & $\operatorname{Max}$ \\
\hline opt -00 & 256 & 397,12 & 1735 & 1 & 267,10 & 4015 & 42 & 2590,26 & 173943 \\
\hline opt -os & 2 & 224,25 & 1021 & 1 & 143,51 & 2882 & 23 & 1516,61 & 82968 \\
\hline opt $-O z$ & 2 & 216,97 & 980 & 1 & 137,11 & 2621 & 22 & 1306,25 & 81426 \\
\hline \multirow[t]{2}{*}{ Best_10 } & 2 & 220,42 & 1017 & 1 & 137,65 & 2542 & 21 & 1290,92 & 86584 \\
\hline & \multicolumn{3}{|c|}{ Funções-Coremark } & \multicolumn{3}{|c|}{ Funções-Mibench } & \multicolumn{3}{|c|}{ LLVM Suíte (Teste) } \\
\hline opt -00 & 48 & 216,41 & 6147 & 428 & 1390,26 & 21525 & 43 & 2064,32 & 12935 \\
\hline opt -Os & 17 & 141,21 & 3407 & 152 & 697,17 & 7843 & 15 & 1370,03 & 8430 \\
\hline opt $-O z$ & 17 & 117,88 & 3407 & 133 & 689,63 & 7879 & 18 & 1053,32 & 6803 \\
\hline Best_10 & 16 & 112,60 & 3415 & 144 & 709,92 & 10232 & 15 & 1032,34 & 7133 \\
\hline
\end{tabular}

Tabela 2. Estatísticas dos conjuntos de dados da classe Embarcados.

\begin{tabular}{lrrrrrr}
\hline & \multicolumn{3}{c}{ Coremark-Pro } & \multicolumn{3}{c}{ Mibench } \\
\hline Instruções & Min & Média & Max & Min & Média & Max \\
\hline opt -o0 & 1488 & 7688 & 23334 & 93 & 23008 & 276055 \\
opt -Os & 842 & 4912 & 14279 & 39 & 12805 & 114317 \\
opt_-Oz & 709 & 4102 & 12222 & 39 & 11822 & 113096 \\
Best_10 & 696 & 3966 & 12789 & 39 & 11993 & 141879 \\
\hline
\end{tabular}


Módulo Extrator de Funções. A fim de se construir arquivos compiláveis contendo funções individuais de uma aplicação, utilizamos a ferramenta $l 1 \mathrm{vm-extract} \mathrm{da} \mathrm{in-}$ fraestrutura LLVM. Esta ferramenta tem como argumentos um programa em codificação IR e o nome da função que se deseja coletar. Tendo em vista que algumas aplicações possuem dezenas de definições de funções, como o caso da aplicação Mibench-c jpg, implementamos um passe LLVM para coleta de nomes e automatização do processo. A Figura 1 descreve a configuração utilizada para construção dos dados da classe Funções.

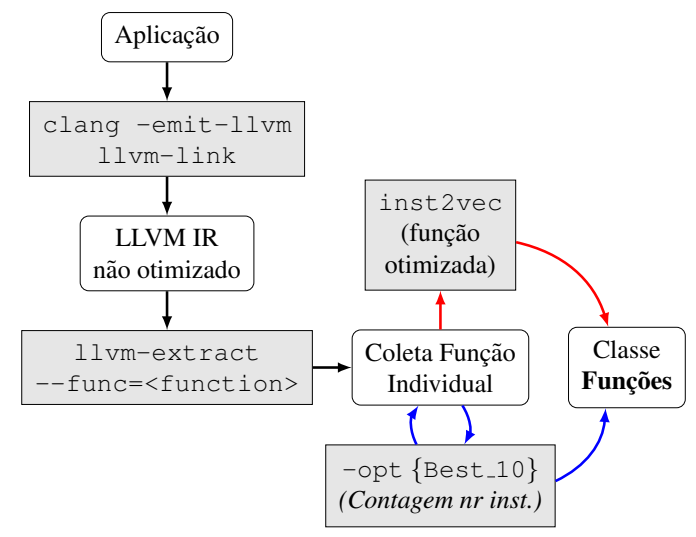

Figura 1. Configuração utilizada para geração de arquivos com funções. A ferramenta $1 \mathrm{lvm}$-extract retorna arquivos compiláveis contendo a definição da função especificada.

Representação de Código. Devido ao sucesso do uso de word-embeddings, vetores densos de alta dimensão, para Processamento de Linguagem Natural, utilizamos a ferramenta inst $2 \mathrm{vec} 6$, que produz embeddings para instruções LLVM de um vocabulário formado por 8564 vetores de 200 dimensões. Para nossos experimentos, a codificação de cada aplicação corresponde a 300 vetores inst2vec. Caso a aplicação contenha um número de instruções inferior a este valor, completamos com vetores de inteiros que serão posteriormente ignorados durante o treinamento pela Rede Neural Artificial por uma camada masking. Utilizamos esta abordagem por duas razões: (1)- para que os conjuntos de dados possam ser totalmente carregados na memória e acelerar o treinamento, tendo em vista que foram gerados modelos de predição para diferentes conjuntos de dados, a utilização de geradores para carregar os dados durante o treinamento não possibilitaria a realização desta pesquisa em tempo hábil; (2)- como mostra a Tabela 1, a média de instruções (opt - O0) dos programas Angha15 e AnghaW, principal foco de análise desta pesquisa, não esta distante do valor escolhido, tornando a perda de informações pouco discrepante.

Sequências de Otimização. Foram construídas 10 sequências de otimização seguindo a metodologia proposta por Silva et al [Silva et al. 2021], utilizando algoritmos de aprendizagem de máquina e as 15.000 maiores funções Angha. Denotamos este conjunto de sequências por Best_10. Dado um programa não otimizado $P_{\text {noopt }}$, existe ao menos uma

\footnotetext{
${ }^{6}$ https://github.com/spcl/ncc
} 
sequência $S_{i} \in$ Best_10 que possui alta probabilidade de ser melhor ou igual à opt - 0 ou opt -Os quando aplicada à $P_{\text {noopt }}$.

As sequências de Best_10 são compostas por 25 passos de otimização, com sequências com tamanho variando no intervalo de inteiros $[9,19]$. Todos os conjuntos de benchmarks descritos foram compilados com as sequências Best_10. A Tabela 3 indica a quantidade de amostras de cada conjunto utilizado para treinamento e teste.

Tabela 3. Número de amostras dos conjuntos compilados com Best_10.

\begin{tabular}{llllll}
\hline & & LLVM_Treino & Angha15 & AnghaW & \\
\hline \#Treino & & 1.750 & 20.000 & 20.000 & \\
\hline & LLVM_Teste & Coremark-f & Mibench-f & Coremark & Mibench \\
\hline \#Teste & 400 & 3.000 & 3.000 & 90 & 190 \\
\hline
\end{tabular}

Arquitetura e Hiperparâmetros Nossa proposta consiste em utilizar arquiteturas LS TM e embeddings para codificação de programas e sequências. A Rede Neural Recorrente consiste em um modelo com duas entradas, onde uma entrada recebe a aplicação não otimizada codificada em vetores inst $2 \mathrm{vec}$, enquanto a outra recebe uma sequência de transformações codificada em inteiros no intervalo $[1,19]$. Apesar de possível o processamento das sequências como inteiros, esta metodologia não é adequada, pois, não indica as relações de dependência entre as transformações da sequência. Dessa forma, uma camada embedding recebe as sequências de inteiros e as transformam em vetores densos de 32 dimensões. Este processo ocorre durante o treinamento.

Após o processamento da aplicação e da sequência de transformações por camadas LSTM, os vetores são então concatenados, formando um único vetor. Após a concatenação, aplicamos uma Normalização de Lotes, com o objetivo de estabilizar a aprendizagem e acelerar o treinamento. O vetor resultante serve como entrada para uma Rede Densa de duas camadas, cujo a saída é a estimativa do número de instruções da aplicação otimizada com a sequência processada. A Figura 2 ilustra nosso modelo de Rede Neural Artificial.

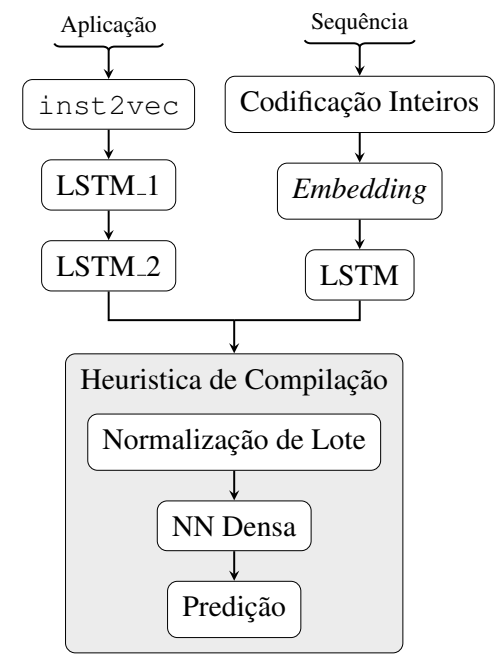

Figura 2. Modelo LSTM para predição de tamanho de código. 
Todas as camadas do modelo utilizam a função de ativação tanh, com exceção da última camada, cujo a função é linear. O modelo foi testado com a função ReLU, porém, esta função de ativação ocasionou explosões de gradiente. A função de erro MAPE (Mean Absolute Percentage Error) 1 foi utilizada para calcular a acurácia, pois, sua saída é uma porcentagem e não depende do intervalo de valores $y$, portanto pode ser utilizada para comparação entre conjuntos de dados distintos.

$$
\operatorname{MAPE}(y, \hat{y})=100 * \frac{\sum_{i=1}^{n}|y-\hat{y}|}{n}
$$

Além da métrica MAPE, avaliamos os conjuntos perante as métricas de correlação Spearman e Pearson, métricas que indicam relações lineares e monotônicas entre os dados. Os modelos foram ajustados para execução por 500 épocas, com critério de parada EarlyStopping definido para 20 épocas. O treinamento se deu em um hardware Intel(R) Xeon(R) CPU @ 2.20GHz, 4 núcleos, 26GB de RAM e GPU Tesla P100.

Buscamos investigar as seguintes questões pertinentes ao nosso sistema de compilação preditiva e aos conjuntos de dados avaliados:

- Qual a acurácia de nosso modelo nos diversos domínios de programas avaliados?

- Em qual cenário AngaBench é mais preciso?

- Quais os aspectos passíveis de melhorias do nosso preditor?

- Benchmarks sintéticos podem incrementar a acurácia do modelo quando utilizados em conjunto com aplicações reais?

\section{Resultados e Discussão}

Iniciamos mostrando o melhor erro médio entre 3 execuções do nosso preditor em cada conjunto de testes. A Figura 3 expõe a verdade com relação a classe de programas Embarcados. Nosso preditor não foi capaz de realizar estimativas com baixos erros. Com relação aos programas selecionados para teste da suíte LLVM, é notável que o conjunto de funções Angha15 apresenta o maior erro, no mais, os outros conjuntos apresentam performance similar. AnghaW e AnghaW+LLVM apresentaram o menor erro médio para funções Coremark-Pro, 20,35\% e 20,75\% respectivamente, seguido por LLVM com $25,20 \%$. Os erros tendem a ser maiores para funções Mibench, uma vez que, estas funções formam arquivos maiores, como indicado na Tabela 1 , $\mathrm{O}$ menor erro médio para este conjunto foi obtido com a suíte de treinamento LLVM com um valor MAPE de aproximadamente $42,06 \%$.

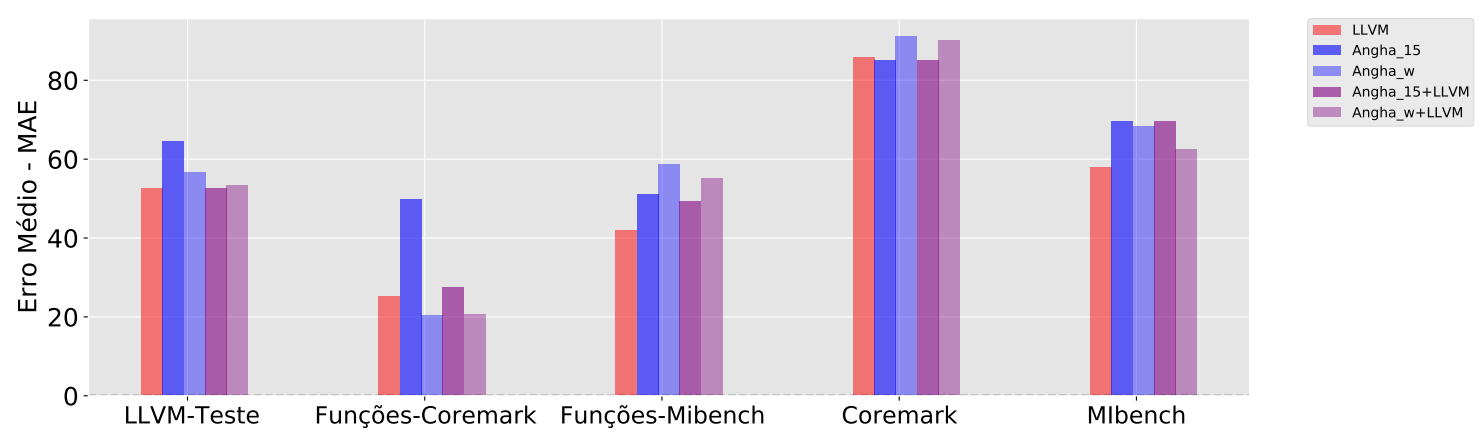

Figura 3. Melhor erro médio de 3 execuções para cada conjunto de testes. 
Diferentemente, a Tabela 4 sumariza os resultados dos modelos com a melhor média em cada classe de programas. Os resultados indicam maior precisão de AnghaW com relação a Angha15, possuindo acurácia próxima à LLVM. Por outro lado, as combinações Angha15+LLVM e AnghaW+LLVM não proporcionaram ganhos com relação à LLVM, mas em sua maioria, incrementaram o erro.

Tabela 4. MAPE do modelo com melhor média da predição de tamanho de código

\begin{tabular}{llll}
\hline $\begin{array}{l}\text { Conjunto de } \\
\text { Dados }\end{array}$ & Funções & Embarcados & LLVM_Teste \\
\hline LLVM_Treino & $39,15 \%$ & $72,17 \%$ & $52,60 \%$ \\
Angha15 & $63,61 \%$ & $78,46 \%$ & $64,57 \%$ \\
AnghaW & $39,76 \%$ & $79,76 \%$ & $56,78 \%$ \\
Angha15+LLVM & $41,42 \%$ & $78,46 \%$ & $52,49 \%$ \\
AnghaW+LLVM & $39,06 \%$ & $76,40 \%$ & $53,39 \%$ \\
\hline
\end{tabular}

Os resultados, em parte, podem ser justificados pela distribuição dos alvos. As Figuras $4 \mathrm{e} 5$ expõe a frequência relativa dos valores reais, i.e., a quantidade de instruções das amostras compiladas com sequências Best_10. Funções e benchmarks Angha tendem a ser arquivos menores e, ao observarmos (a), (b) e (c) é notável que LLVM+Angha 15 e LLVM+AnghaW deslocariam a frequência para valores menores, o que pode explicar a ausência de melhorias.

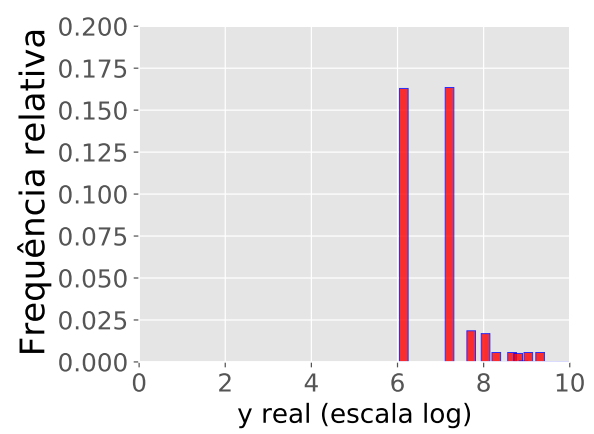

(a) LLVM

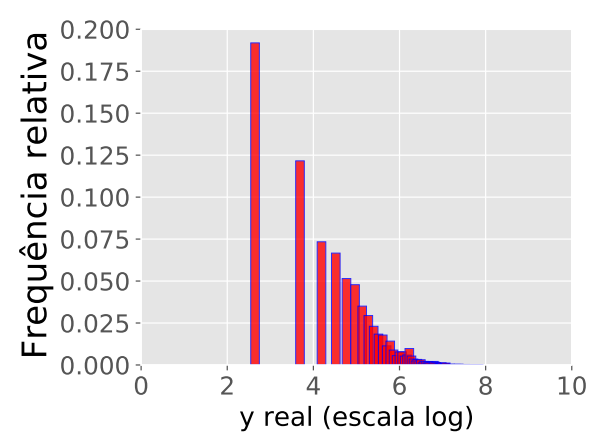

(c) AnghaW

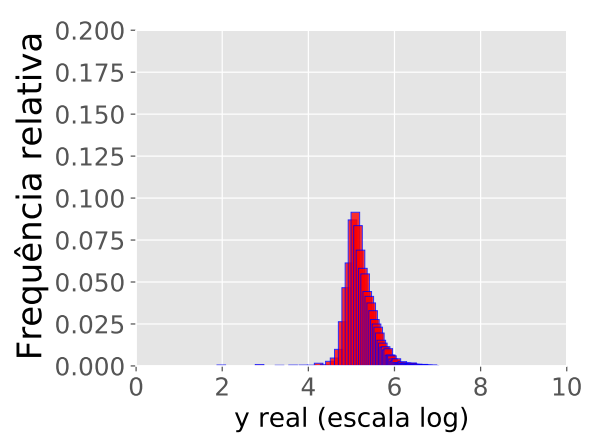

(b) Angha15

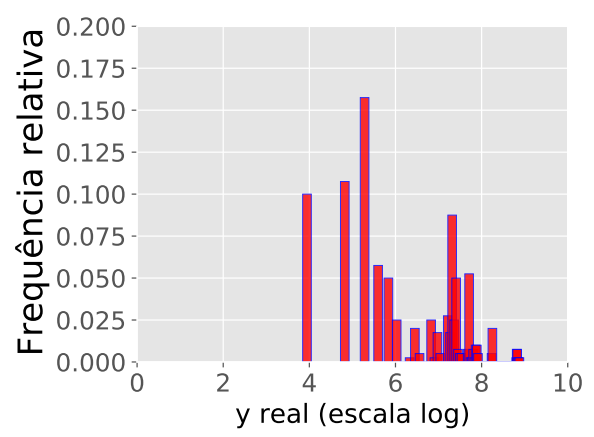

(d) LLVM_Teste

Figura 4. Frequências relativas dos alvos (compilação com Best_10) 


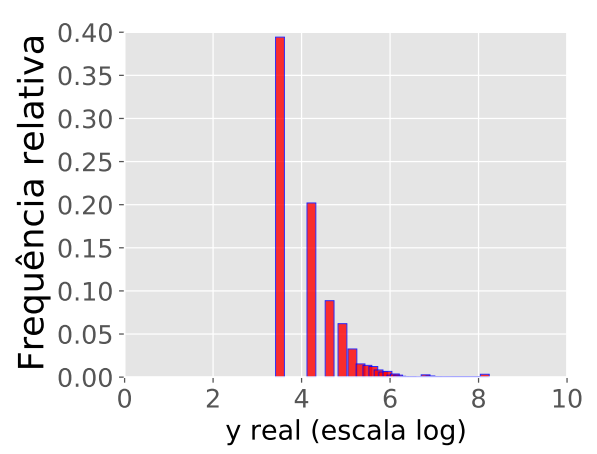

(a) Coremark-f

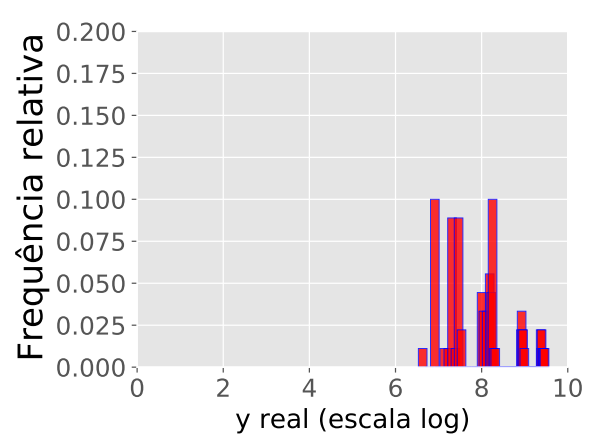

(c) Coremark

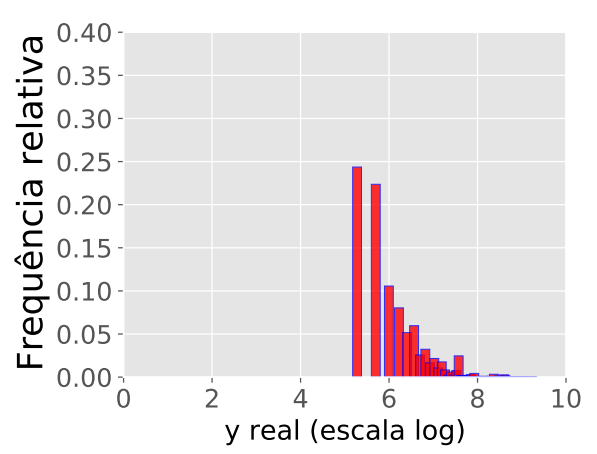

(b) Mibench-f

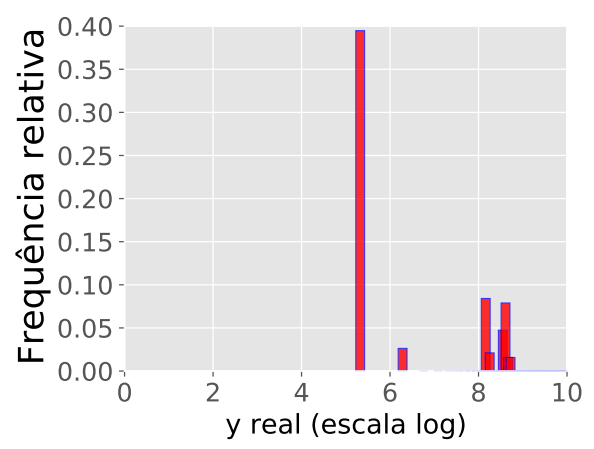

(d) Mibench

Figura 5. Frequências relativas dos alvos (compilação com Best_10)

Tabela 5. Coeficientes de Pearson (CP) e Spearman (CS) dos modelos com meIhor performance em cada conjunto individual de testes. A similaridade é calculada entre o valor real e o valor previsto

\begin{tabular}{l|ll|ll|ll|ll|ll}
\hline & \multicolumn{2}{|l|}{ LLVM_Teste } & \multicolumn{2}{l|}{ Coremark-f } & \multicolumn{2}{|l|}{ Mibench-f } & \multicolumn{2}{l|}{ Coremark } & \multicolumn{2}{l}{ Mibench } \\
\cline { 2 - 11 } & CP & CS & CP & CS & CP & CS & CP & CS & CP & CS \\
\hline LLVM_Treino & 0,34 & 0,37 & 0,44 & 0,91 & 0,04 & 0,1 & $-0,00$ & 0,04 & 0,12 & 0,34 \\
\hline Angha15 & 0,22 & 0,34 & 0,14 & 0,21 & 0,26 & 0,19 & 0,02 & 0,07 & 0,46 & 0,57 \\
AnghaW & 0,24 & 0,27 & 0,39 & 0,92 & $-0,05$ & $-0,01$ & $-0,20$ & $-0,24$ & $-0,19$ & 0,03 \\
\hline Angha15+LLVM & 0,35 & 0,58 & 0,35 & 0,88 & 0,12 & 0,12 & 0,02 & 0,07 & 0,46 & 0,57 \\
AnghaW+LLVM & 0,24 & 0,27 & 0,39 & 0,92 & 0,32 & 0,90 & 0,01 & 0,04 & $-0,36$ & $-0,33$ \\
\hline
\end{tabular}

Como já discutido, a codificação dos programas em uma representação de tamanho fixo pode prejudicar a qualidade dos dados, pois, pode levar a perda de informações. Ao observarmos as Tabelas 1 e 2, a média dos benchmarks Angha15 e AnghaW são 397, 12 e 267, 10 instruções, respectivamente. Portanto, a escolha do valor de truncamento correspondente a 300 vetores parece razoável. No entanto, isso pode prejudicar a aprendizagem do modelo em aplicações maiores, como é o caso das amostras LLVM e Embarcados, que contemplam aplicações com milhares de instruções. O fato da acurácia do conjunto LLVM (a) não ser satisfatória com relação ao conjunto Embarcados $(\mathrm{g}, \mathrm{h})$ pode estar relacionado a codificação já citada. Portanto, uma representação que seja capaz de codificar todas as propriedades dos benchmarks e seja invariante com relação ao tamanho do código pode resultar em melhorias. 
Por fim, complementamos nossa avaliação calculando os coeficientes de similaridade de Pearson e Spearman, indicados na Tabela 5. Valores de coeficiente de Pearson próximos de 1,00 indicam uma correlação linear muito forte entre os dados, por outro lado, valores de coeficiente de Spearman próximos de 1 indicam uma correlação monotônica muito forte. Valores positivos de Spearman, como no caso de funções Coremark, indicam que, conforme os valores reais das amostras crescem, os valores preditos pelo sistema também tendem a crescer.

\section{Conclusões e Trabalhos Futuros}

Neste trabalho, apresentamos um sistema de predição de tamanho de código para sequências de otimização, utilizando redes LSTM como heurística. Estudamos o comportamento de programas sintéticos AnghaBench em comparação com benchmarks reais. Os resultados indicam que, em alguns casos, benchmarks sintéticos apresentam performance similar a programas reais, mas não resultam em melhorias de acurácia quando utilizados em conjunto com benchmarks LLVM.

A análise dos conjuntos de dados, juntamente com o modelo de compilação preditiva nos leva a crer que, as limitações de acurácia podem ser parcialmente atribuídas aos benchmarks escolhidos, que não contemplam satisfatoriamente todos os domínios selecionados para avaliação, e a forma como a codificação das aplicações foi realizada. Acreditamos que o tratamento de cada um desses problemas pode levar em melhorias na acurácia. Os próximos passos incluem mitigar estas questões, utilizando representações de grafos (ASTs, GFCs) que podem enriquecer o modelo de aprendizagem com informações sobre dependência de dados e controle, juntamente com maiores conjuntos de dados.

\section{Referências}

Ashouri, A. H., Killian, W., Cavazos, J., et al. (2018). A Survey on Compiler Autotuning Using Machine Learning. ACM Comput. Surv., 51(5).

Barany, G. (2018a). Finding Missed Compiler Optimizations by Differential Testing. In Proceedings of the 27th International Conference on Compiler Construction, CC 2018, page 82-92, New York, NY, USA. Association for Computing Machinery.

Barany, G. (2018b). Liveness-driven random program generation. In Fioravanti, F. and Gallagher, J. P., editors, Logic-Based Program Synthesis and Transformation, pages 112-127, Cham. Springer International Publishing.

Cummins, C., Petoumenos, P., Murray, A., et al. (2018). Compiler Fuzzing through Deep Learning. In Proceedings of the 27th ACM SIGSOFT International Symposium on Software Testing and Analysis, ISSTA 2018, page 95-105, New York, NY, USA. Association for Computing Machinery.

Cummins, C., Petoumenos, P., Wang, Z., et al. (2017). Synthesizing Benchmarks for Predictive Modeling. In Proceedings of the 2017 International Symposium on Code Generation and Optimization, CGO '17, page 86-99, Austin, USA. IEEE Press.

da Silva, A. F., Kind, B. C., de Souza Magalhães, J. W., et al. (2021). ANGHABENCH: A Suite with One Million Compilable C Benchmarks for Code-Size Reduction. In 2021 IEEE/ACM International Symposium on Code Generation and Optimization (CGO), pages 378-390, Seoul, South Korea. IEEE Computer Society. 
Goens, A., Brauckmann, A., Ertel, S., et al. (2019). A Case Study on Machine Learning for Synthesizing Benchmarks. In Proceedings of the 3rd ACM SIGPLAN International Workshop on Machine Learning and Programming Languages, MAPL 2019, page 38-46, New York, NY, USA. Association for Computing Machinery.

Hashimoto, A. and Ishiura, N. (2016). Detecting Arithmetic Optimization Opportunities for C Compilers by Randomly Generated Equivalent Programs. IPSJ Transactions on System LSI Design Methodology, 9:21-29.

Heo, K., Lee, W., Pashakhanloo, P., et al. (2018). Effective Program Debloating via Reinforcement Learning. In Proceedings of the 2018 ACM SIGSAC Conference on Computer and Communications Security, CCS '18, page 380-394, New York, NY, USA. Association for Computing Machinery.

Hochreiter, S. and Schmidhuber, J. (1997). Long Short-Term Memory. Neural Comput., 9(8):1735-1780.

Huang, Q., Haj-Ali, A., Moses, W., et al. (2020). AutoPhase: Juggling HLS Phase Orderings in Random Forests with Deep Reinforcement Learning.

Lattner, C. and Adve, V. (2004). LLVM: A Compilation Framework for Lifelong Program Analysis and Transformation. In $C G O$, pages 75-88, San Jose, CA, USA.

Otter, D. W., Medina, J. R., and Kalita, J. K. (2021). A Survey of the Usages of Deep Learning for Natural Language Processing. IEEE Transactions on Neural Networks and Learning Systems, 32(2):604-624.

Rocha, R. C. O., Petoumenos, P., Wang, Z., Cole, M., and Leather, H. (2019). Function merging by sequence alignment. In 2019 IEEE/ACM International Symposium on Code Generation and Optimization (CGO), pages 149-163.

Rocha, R. C. O., Petoumenos, P., Wang, Z., Cole, M., and Leather, H. (2020). Effective function merging in the ssa form. In Proceedings of the 41 st ACM SIGPLAN Conference on Programming Language Design and Implementation, PLDI 2020, page 854-868, New York, NY, USA. Association for Computing Machinery.

Silva, A. F. d., de Lima, B. N. B., and Pereira, F. M. Q. a. (2021). Exploring the Space of Optimization Sequences for Code-Size Reduction: Insights and Tools. In Proceedings of the 30th ACM SIGPLAN International Conference on Compiler Construction, CC 2021, page 47-58, New York, NY, USA. Association for Computing Machinery.

Trofin, M., Qian, Y., Brevdo, E., et al. (2021). MLGO: a Machine Learning Guided Compiler Optimizations Framework.

Wei, X.-S., Wu, J., and Cui, Q. (2019). Deep Learning for Fine-Grained Image Analysis: A Survey.

Yang, X., Chen, Y., Eide, E., et al. (2011). Finding and Understanding Bugs in C Compilers. SIGPLAN Not., 46(6):283-294.

Zhang, S., Yao, L., Sun, A., et al. (2019). Deep Learning Based Recommender System: A Survey and New Perspectives. ACM Computing Surveys, 52(1). 